Supporting Information

\title{
Programmable Polymeric Microneedles for Combined Chemotherapy and Antioxidative Treatment of Rheumatoid Arthritis
}

\author{
Chaoxiong Wu, Jiale Cheng, Wei Li, Lingzhi Yang*†, Haifeng Dong*, and Xueji \\ Zhang
}

Marshall Laboratory of Biomedical Engineering Research Center for Biosensor and Nanotheranostic, School of Biomedical Engineering, Health Science Center, Shenzhen University, Guangdong 518060, P.R.China 
A
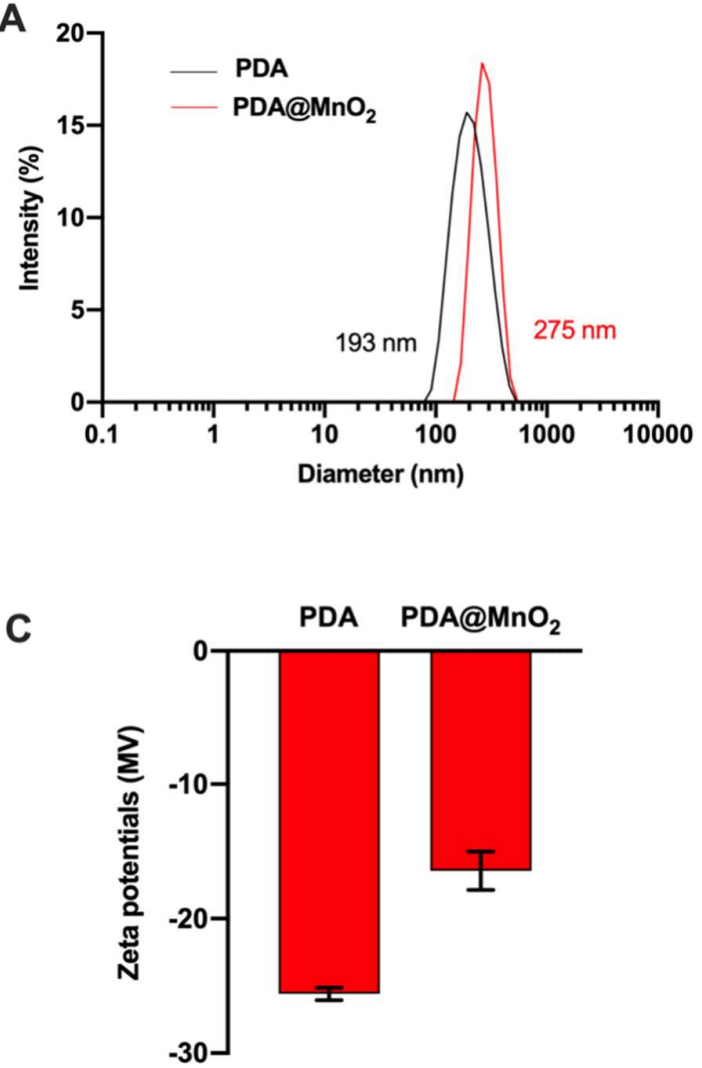

B

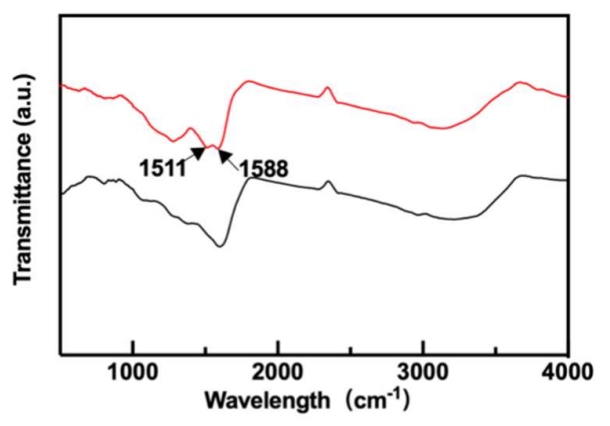

Figure S1. DLS (A), FTIR spectra (B) and ZETA potential (C) of PDA and PDA@ $\mathrm{MnO}_{2}$. 


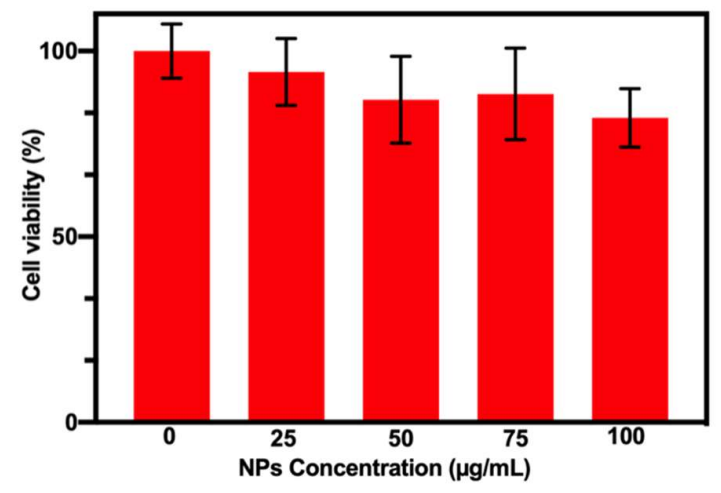

Figure S2. Cytotoxicity of PDA@ $\mathrm{MnO}_{2}$ incubating with MC3T3-E1 cells. 


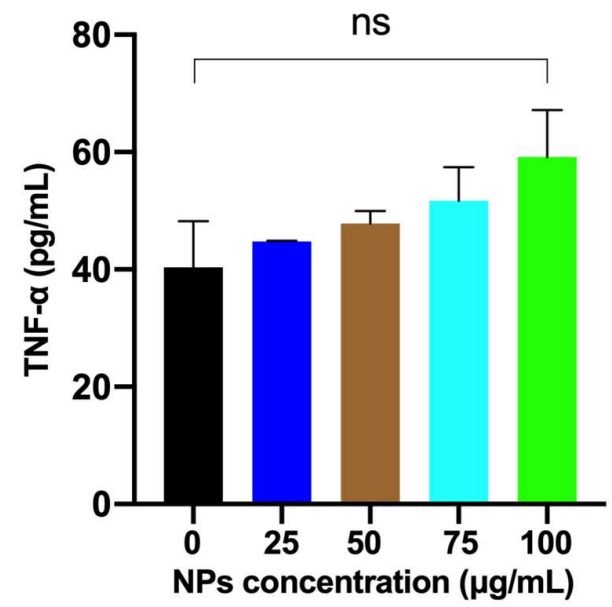

Figure S3. The LPS-treated RAW264.7 cells of TNF- $\alpha$ level with different concentration of NPs. 

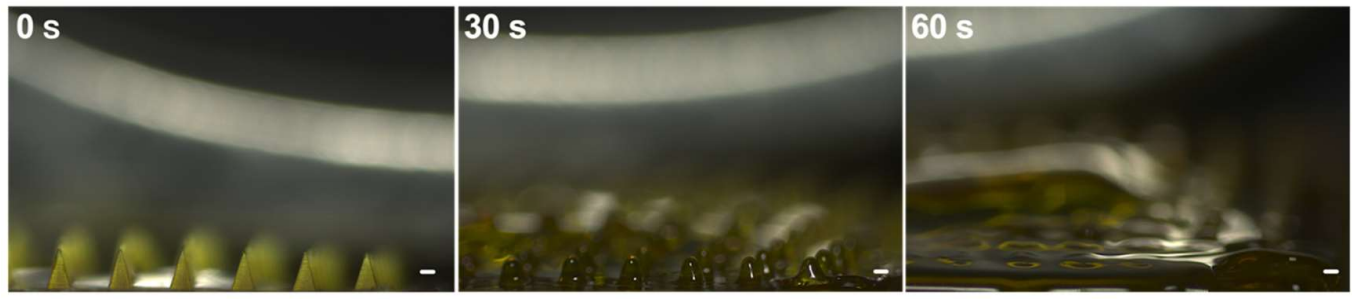

Figure S4. The optical microscopy images of PVP MNs patch from side view before and after insertion for 30,60s into the agarose in vitro. The scale bar is $500 \mu \mathrm{m}$. 

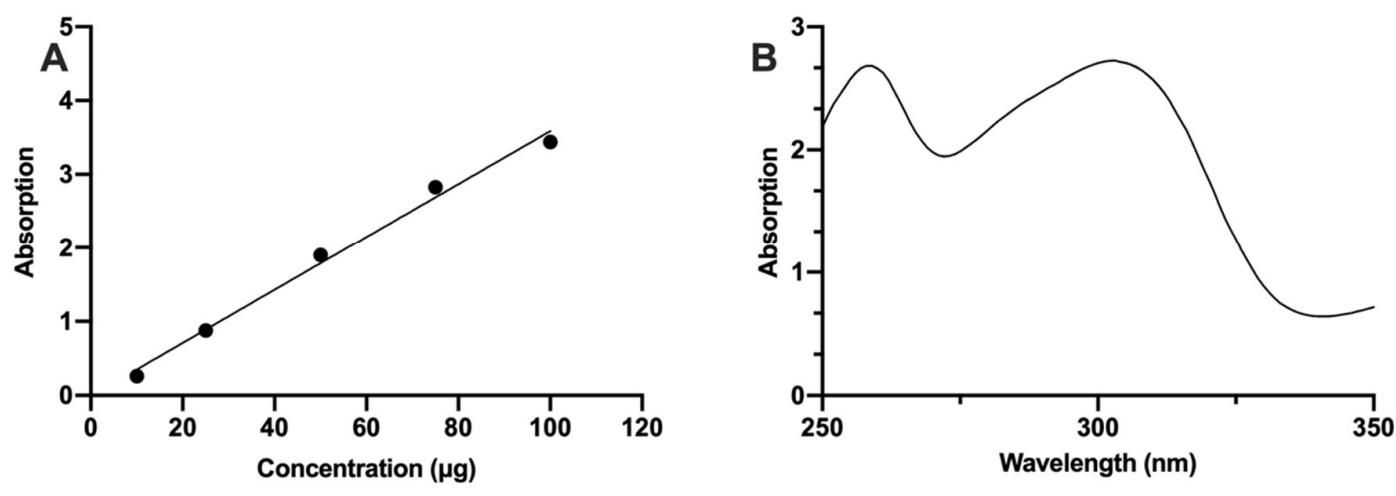

Figure S5. (A) The standard curve of MTX calculated by UV-vis absorption. (B) The UV-vis absorption of MTX in the MNs. 


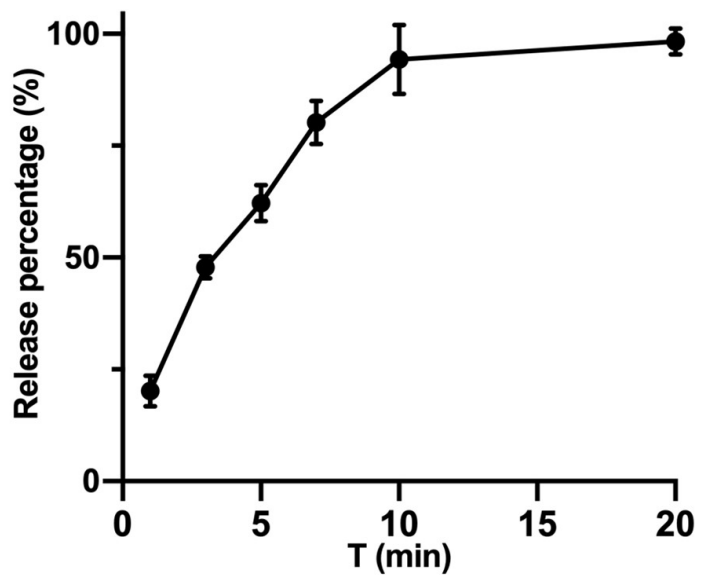

Figure S6. Cumulative percent drug released at 1, 3, 5, 7, 10, 20 min. Data are presented as mean $\pm \operatorname{SD}(n=3)$. 